DOI: $10.17516 / 1998-2836-0250$

УДК 533.15:541.12

\title{
Potential Landscape of a Probe Penetrant Particle for Fast Estimating of Silica Diffusion Properties
}

\author{
Sergey V. Kukhtetskiy*, \\ Elena V. Fomenko and Elena S. Rogovenko \\ Institute of Chemistry and Chemical Technology SB RAS \\ Krasnoyarsk, Russian Federation
}

Received 12.05.2021, received in revised form 22.07.2021, accepted 02.09.2021

\begin{abstract}
Successful search for optimal molecular structures of membrane materials requires efficient algorithms for assessing their diffusion properties. It is shown in this work that the potential landscape of a probe penetrating particle, a component that passes through the membrane during gas separation, is suitable for solving such problems. A number of indicators are considered that can be easily calculated from potential landscapes of specific models of silicate materials, both not related to the topology of the potential landscape (global minimum, voxel energy distribution), and depending on it (percolation cluster). A good correlation of these indicators with the corresponding diffusion coefficients is shown.
\end{abstract}

Keywords: silica, helium, solubility site, diffusion, probe particle, potential landscape, voxel.

Citation: Kukhtetskiy S. V., Fomenko E. V. and Rogovenko E. S. Potential landscape of a probe penetrant particle for fast estimating of silica diffusion properties, J. Sib. Fed. Univ. Chem., 2021, 14(3), 406-417. DOI: 10.17516/1998-2836-0250

(C) Siberian Federal University. All rights reserved

This work is licensed under a Creative Commons Attribution-NonCommercial 4.0 International License (CC BY-NC 4.0).

* Corresponding author E-mail address: kswrk@mail.ru 


\title{
Использование потенциального ландшафта пробной частицы пенетранта для быстрой оценки диффузионных свойств кремнезема
}

\author{
С. В. Кухтецкий, Е. В. Фоменко, Е. С. Роговенко \\ Институт химии и химической технологии \\ ФИЦ КНЦ СО РАН \\ Российская Федераиия, Красноярск
}

\begin{abstract}
Аннотация. Для успешного поиска оптимальных молекулярных структур мембранных материалов необходимы эффективные алгоритмы оценки их диффузионных свойств. В работе показано, что потенциальный ландшафт пробной частицы пенетранта - компонента, который в процессе газоразделения проходит через мембрану, является подходящим для решения такого рода задач. Рассмотрен ряд показателей, легко вычисляемых по потенциальным ландшафтам конкретных моделей силикатных материалов, как не связанных с топологией потенциального ландшафта (глобальный минимум, распределение вокселей по энергии), так и зависящих от нее (перколяционный кластер). Представлена хорошая корреляция этих показателей с соответствующими коэффициентами диффузии.
\end{abstract}

Ключевые слова: кремнезем, гелий, сайт растворимости, диффузия, пробная частица, потенциальный ландшафт, воксел.

Цитирование: Кухтецкий, С. В. Использование потенциального ландшафта пробной частицы пенетранта для быстрой оценки диффузионных свойств кремнезема / С. В. Кухтецкий, Е. В. Фоменко, Е. С. Роговенко // Журн. Сиб. федер. ун-та. Химия, 2021, 14(3). С. 406-417. DOI: 10.17516/1998-2836-0250

\section{Введение}

Материалы на основе непористых силикатных стекол считаются перспективными при создании высокоселективных газоразделительных мембран для извлечения легких газов (гелий, водород) [1], так как характеризуются высокими значениями коэффициентов разделения смесей (например, при $400{ }^{\circ} \mathrm{C} \alpha \mathrm{He} / \mathrm{CH}_{4}=10^{6}$ [2], $\alpha \mathrm{He} / \mathrm{N}_{2}=10^{5}$ [3]). Однако по уровню проницаемости силикатные стекла уступают хорошо изученному классу мембран на основе полимеров $[4,5]$.

В связи с тем, что процессы растворения и диффузии газов в силикатных стеклах в значительной степени определяются геометрией свободного пространства материала, одним из возможных путей увеличения их проницаемости при сохранении высокой селективности для конкретных газовых смесей может быть управляемая модификация топологии пространственной сети стеклообразной матрицы. В настоящее время в связи с быстрым развитием программно-аппаратных средств первичный поиск и исследование диффузионных свойств мембранных материалов с определенной модифицированной структурой можно осуществлять теоретически. Компьютерные молекулярные модели кварцевого стекла, пригодные для таких целей, представлены в работах [6-8]. Показано, что основные структурные параметры

$$
-407-
$$


ближнего порядка и плотность как важнейший структурный параметр среднего порядка [6, 7] для полученных модельных образцов, а также результаты молекулярно-динамического моделирования процесса диффузии гелия в них [8] хорошо совпадают с экспериментальными значениями.

Оценка диффузионных свойств моделей стекол в работе [8] проводилась путем непосредственного моделирования миграции атомов гелия в матрице кремнезема методом молекулярной динамики. В силу своей предельной детальности этот метод вполне эффективен для решения диффузионных задач, но достаточно трудоемок с вычислительной точки зрения. Использование молекулярной динамики для газов с бо́льшими кинетическими диаметрами (водород, неон) или при низких температурах связано с существенным увеличением времени моделирования из-за уменьшения частоты скачков атомов пенетранта (компонент, который в процессе газоразделения проходит через мембрану) из одной полости свободного пространства матрицы мембраны в другую. Подавляющее количество вычислительных ресурсов расходуется на отслеживание колебаний атома внутри полостей материала, что не позволяет за приемлемое время получить статистически представительные данные по их миграции между полостями, необходимые для корректного вычисления коэффициентов диффузии.

Для эффективной оценки диффузионных характеристик (растворение и диффузия газов) мембранных материалов необходима разработка более простых методик, базирующихся непосредственно на молекулярной структуре самого материала и не требующих молекулярнодинамического моделирования миграции пробных атомов пенетранта в матрице мембраны.

Растворение и диффузия газов, не реагирующих с матрицей, предполагает наличие в ней областей пространства, в которых атомы газов могут размещаться. Совокупность этих областей обычно называется свободным пространством (англ. «free space») и широко используется при анализе процессов растворения и диффузии. В отличие от пористых тел, например, с кнудсеновской диффузией, для которых понятие свободного пространства имеет вполне однозначный геометрический смысл, в применении к непористым материалам этот термин становится неконструктивным. Главная причина заключается в том, что газовая диффузия в непористых материалах имеет активационный характер, т. е. области пространства, доступные атомам пенетранта, зависят от их энергии. В результате геометрия свободного пространства материала очень зависит от типов и энергий атомов мигрирующего газа: для низкоэнергетичных частиц геометрия свободного пространства может быть одна, для высокоэнергетичных - другая, а поскольку все мигрирующие частицы быстро термализуются, то даже для одной и той же частицы геометрия свободного пространства становится довольно неопределенной величиной.

В этой связи более определенным является понятие «энергетический ландшафт» (англ. «energy landscape»), которое широко и успешно используется во многих областях физической химии $[9,10]$. В применении к задаче активированной диффузии газов в силикатных стеклах при температурах, далеких от температуры размягчения, матрицу можно считать «замороженной». Энергетический ландшафт становится низкоразмерным, представляя собой скалярное поле потенциальной энергии подсистемы атомов пенетранта в матрице мембраны. Если внешнее давление газа не слишком велико (порядка атмосферного), то энергетический ландшафт еще сильнее упрощается - он становится трехмерным. Таким образом, энергетический 
ландшафт содержит полную информацию о диффузионных свойствах материала. Однако для практического сравнения мембранных материалов необходима более компактная система показателей, основанных на ландшафтах.

В данной работе представлена методика, разработанная на основании топологического анализа энергетического ландшафта системы «диффундирующий атом - мембранный материал» с последующим расчетом параметров, включающих локальные (глобальный минимум, функция распределения вокселей по энергии) и комплексные показатели (перколяционные кластеры).

\section{Материалы и методика исследования}

Задача оценки диффузионных свойств материала сводится к анализу поведения одной единственной частицы газа в расчетной ячейке. Коэффициент растворимости, например, для гелия в кварцевом стекле составляет величину порядка $10^{-2}$. В этом случае концентрация растворенного газа находится на уровне $10^{-17} \mathrm{~cm}^{-3}$. Размер расчетной ячейки обычно $100 \AA$, среднее количество мигрирующих частиц в объеме ячейки оценивается величиной 0.1 , а вероятность нахождения одновременно двух атомов гелия в расчетной ячейке не превышает 1 \%.

Таким образом, энергетический ландшафт системы представляет собой трехмерное поле потенциальной энергии атома/молекулы пенетранта в расчетной ячейке, заполненной атомами матрицы. Начальное состояние системы - атом пенетранта находится на одной грани системы, конечное - атом находится на противоположной грани. В этом случае координаты реакции и путь системы в процессе «трансформации» представляют собой реальную траекторию мигрирующей частицы в трехмерном пространстве.

Для практического сравнения мембранных материалов требуется некоторая компактная система показателей, основанных на энергетических ландшафтах. Ниже рассмотрены такие показатели, позволяющие количественно сравнивать диффузионные свойства молекулярных моделей материалов. Для оценки эффективности этих показателей были использованы несколько наборов моделей силикатных материалов, различающихся структурой среднего порядка и, соответственно, коэффициентами диффузии:

1. Образцы моделей кварцевого стекла, полученные при одних и тех же условиях, но различающиеся конфигурацией атомов перед началом закалки (образцы s01_1 - s01_6). Образцы

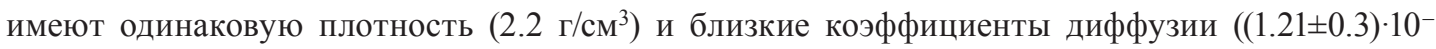
${ }^{6} \mathrm{~cm}^{2} / \mathrm{c}$, при температуре $500 \mathrm{~K}$ [8]). Этот набор образцов был нужен для оценки чувствительности показателей к случайным вариациям атомных конфигураций при близких диффузионных (макроскопических) свойствах образцов.

2. Набор образцов моделей кварцевого стекла с монотонно изменяющейся плотностью

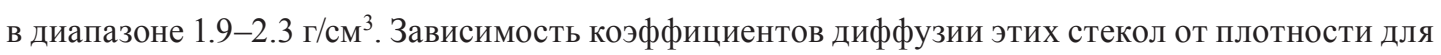
температуры $500 \mathrm{~K}$ описывается следующей регрессионной зависимостью:

$$
\mathrm{D}=722.76 \cdot \exp (-8.939 \cdot \rho)
$$

где $\mathrm{D}$ - коэффициент диффузии, $\mathrm{cm}^{2} / \mathrm{c} ; \rho$ - плотность стекла, г $/ \mathrm{cm}^{3}$.

Этот набор образцов был нужен для оценки корреляции показателей, рассчитанных по энергетическому ландшафту, с коэффициентами диффузии.

$$
-409-
$$


3. Два кристаллических образца с диаметрально противоположными диффузионными свойствами. Первый - кубический кристобалит, второй - чисто кремниевый содалит (pure silica sodalite). Кристобалит имеет низкую плотность, близкую к плотности кварцевого стекла. Все полости свободного пространства кристобалита соединены друг с другом 6-членными кольцами (Si-O). Других проходов в кристобалите нет. В материалах на основе кремнезема 6-членные кольца как раз обеспечивают достаточно высокую проницаемость при высокой селективности по отношению к более крупным молекулам (азот, метан, двуокись углерода и т. п.). Но в то же время, например, при температуре $500 \mathrm{~K}$ кристобалит обладает примерно на два порядка меньшим коэффициентом диффузии, чем кварцевое стекло $\left(1.4 \cdot 10^{-8} \mathrm{~cm}^{2} / \mathrm{c}-\right.$ экстраполяция данных [11] для кристобалита на температуру $500 \mathrm{~K}$ и $1.16 \cdot 10^{-6} \mathrm{~cm}^{2} / \mathrm{c}$ - для плавленного кварцевого стекла [12]). Второй кристаллический образец - модель чисто кремниевого содалита. Экспериментальных данных по диффузии гелия в этом материале в литературе нет. Поэтому коэффициент диффузии гелия в этом образце был рассчитан для температуры $500 \mathrm{~K}$ по методике, описанной в работе [8]. Его значение $2.719 \cdot 10^{-5} \mathrm{~cm}^{2} / \mathrm{c}$, что более чем на порядок превышает коэффициент диффузии кварцевого стекла.

Для каждого образца был построен энергетический ландшафт. Пенетрант - гелий, в качестве потенциала взаимодействия гелия с кислородной подсистемой взят потенциал БорнаМайера, параметризованный в работе [8]. Технически каждый ландшафт представляет собой трехмерную матрицу ячеек (вокселей) 256x256x256, дискретизирующую кубическую расчетную ячейку образца кремнезема. В каждом вокселе - значение потенциала взаимодействия атома гелия с матрицей в соответствующей точке пространства. Для анализа энергетического ландшафта использовано оригинальное программное обеспечение, разработанное в процессе решения задачи.

\section{Анализ и обсуждение полученных результатов}

Задача оценки диффузионных свойств материала сводится к исследованию топологических свойств трехмерных энергетических ландшафтов, построенных для каждого конкретного атома пенетранта, по одному ландшафту на каждый тип диффундирующего атома или молекулы. В данной работе анализ ограничен лишь одним типом атома - гелием. Стереопара изоповерхности энергетического ландшафта для уровня энергии 0.1 эВ показана на рис. 1, а для 0.5 эВ - на рис. 2. Методика расчета энергетического ландшафта описана ниже, для визуализации ландшафта использован свободный пакет VMD (Visual Molecular Dynamics), разработанный в Центре макромолекулярного моделирования и биоинформатики Иллинойского университета в Урбане-Шампейне, США. Стереопары являются скрещенными (для простоты визуализации необходимо левым глазом посмотреть на правую картинку и наоборот). Видно, как критически зависит геометрия свободного пространства от энергии мигрирующих частиц. Кроме этого, свободное пространство кварцевого стекла мало похоже на классические локализованные «сайты растворимости» (англ. «solubility sites»), соединенные «дверьми» (англ. «doorways»), через которые совершают прыжки атомы пенетранта, и скорее похоже на «губку», представляющую собой сеть пересекающихся каналов переменного сечения (рис. 2).

Простые глобальные показатели можно рассчитать без учета топологии энергетического ландшафта. Самый простейший из них - глобальный минимум потенциальной энергии атома 


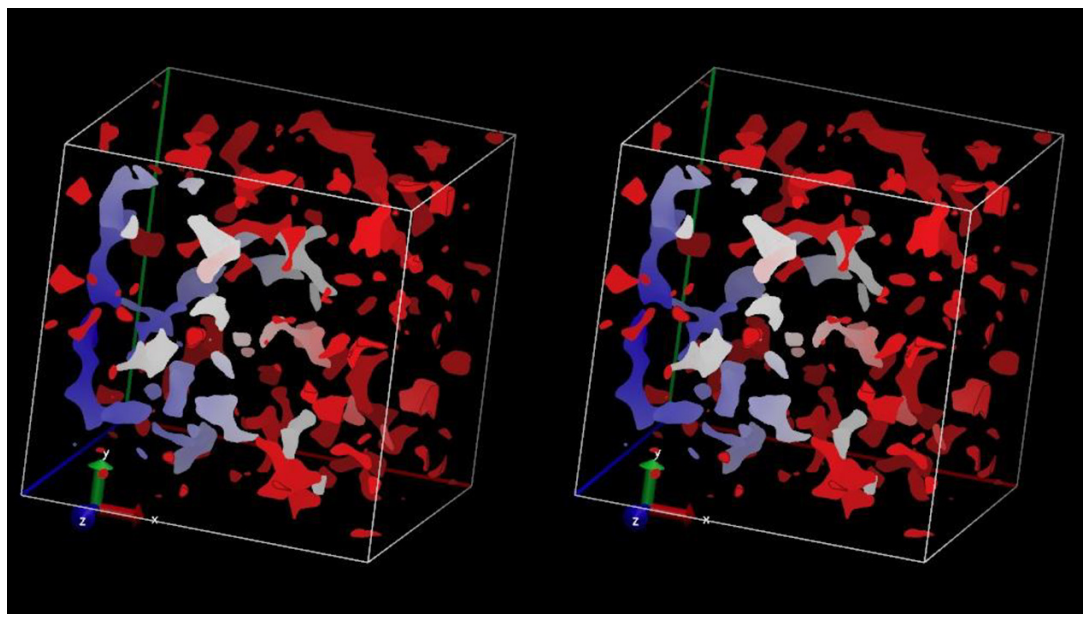

Рис. 1. Перекрестная стереопара изоповерхности (0.1 эВ) энергетического ландшафта кварцевого стекла (образец сr_2.200_6)

Fig. 1. Cross stereopair of the isosurface $(0.1 \mathrm{eV})$ of the energy landscape of quartz glass (sample cr_2.200_6)

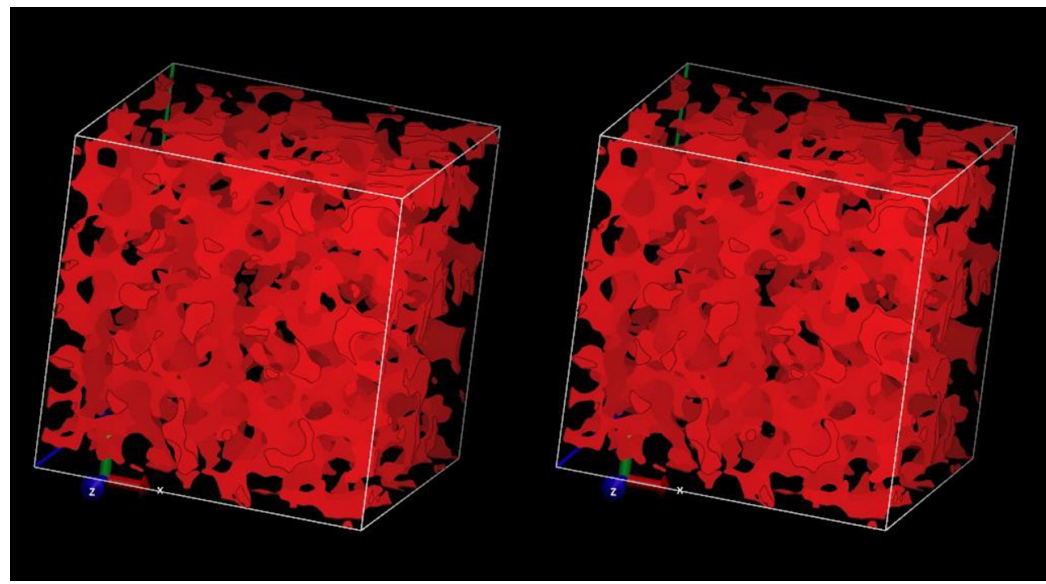

Рис. 2. Перекрестная стереопара изоповерхности (0.5 эВ) энергетического ландшафта кварцевого стекла (образец сr_2.200_6)

Fig. 2. Cross stereopair of the isosurface $(0.5 \mathrm{eV})$ of the energy landscape of quartz glass (sample cr_2.200_6)

гелия в образце. На рис. 3 представлена зависимость глобальных минимумов энергетических ландшафтов образцов от их коэффициентов диффузии, из которой явно видна корреляция этих величин. Регрессионная зависимость степенная:

$$
\mathrm{U}_{\min }=\mathrm{A} \cdot \mathrm{D}^{-\mathrm{B}}
$$

где $\mathrm{U}_{\min }$ - глобальный минимум потенциальной энергии атома гелия в образце, эВ; D - коэффициент диффузии, см ${ }^{2} / \mathrm{c} ; \mathrm{A}=3.861 \cdot 10^{-9}, \mathrm{~B}=0.819$.

Менее удобным, но более наглядным глобальным нетопологическим показателем является функция распределения ячеек (вокселей) энергетического ландшафта по энергии, т. е. 


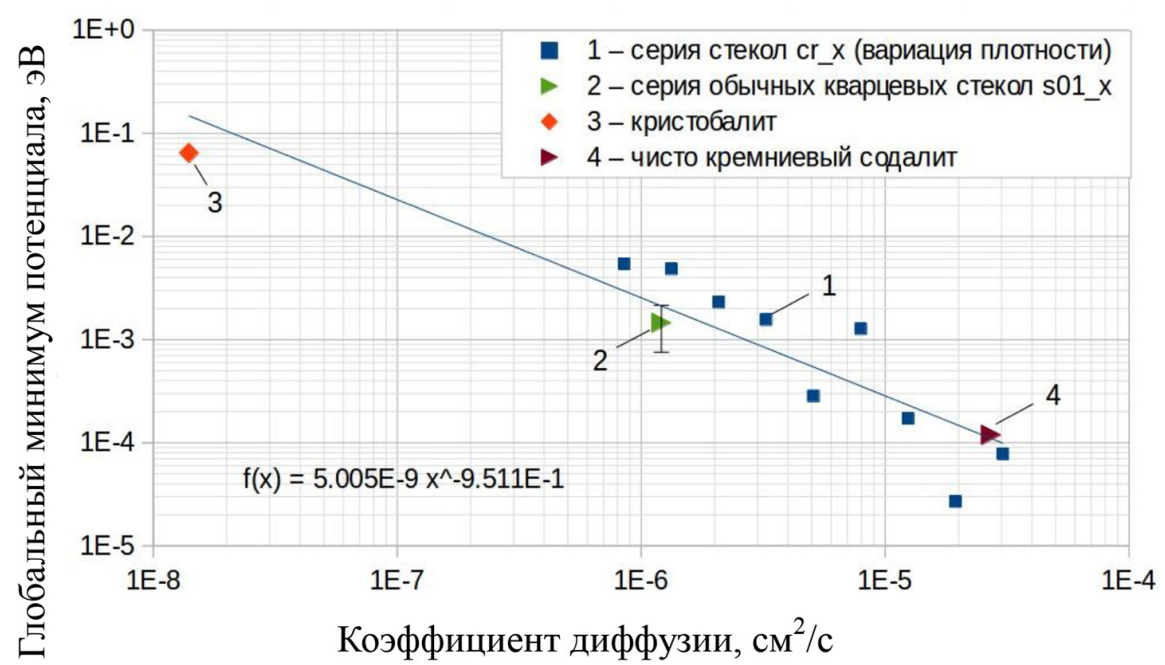

Рис. 3. Зависимость глобальных минимумов энергетических ландшафтов образцов от их коэффициентов диффузии

Fig. 3. Dependence of the global minima of the energy landscapes of the samples on their diffusion coefficients

зависимость суммарного объема вокселей, обладающих заданным уровнем энергии, от энергии. Объемы нормированы на общий объем пространства. Примеры таких функций распределения для нескольких образцов показаны на рис. 4. Нумерация кривых выполнена в порядке возрастания коэффициентов диффузии соответствующих образцов от кристобалита (1), обладающего минимальной проницаемостью, до полностью кремниевого содалита (6), обладающего максимальной газовой проницаемостью. Видно, что коэффициент диффузии

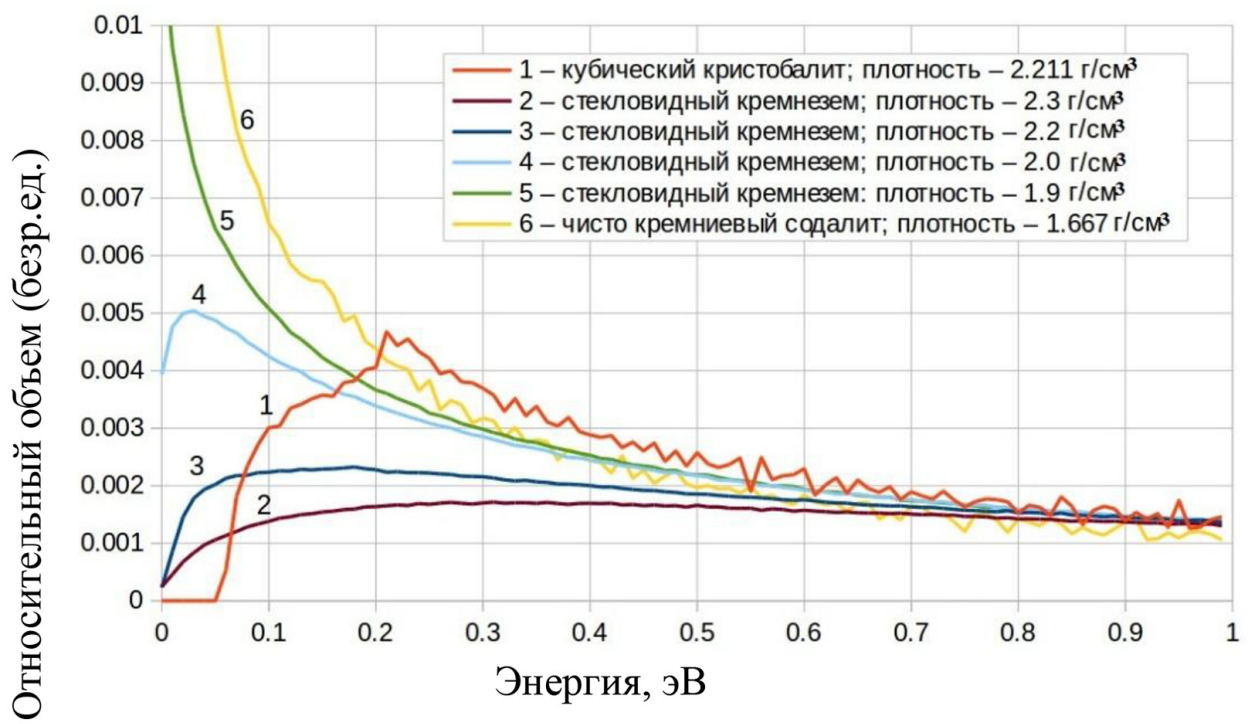

Рис. 4. Нормированные на объем функции распределения вокселей по энергии

Fig. 4. Volume-normalized voxel energy distribution functions 
монотонно возрастает с увеличением доли вокселей энергетического ландшафта с низкой энергией.

Продемонстрированные выше нетопологические показатели вполне хорошо коррелируют с коэффициентом диффузии и могут использоваться как некие полуколичественные индикаторы при поиске нужных структур среднего порядка конструируемого материала. Однако пользоваться ими необходимо с осторожностью из-за одного принципиального недостатка. Эти показатели никак не учитывают топологию энергетического ландшафта и, в принципе, неспособны отражать низкоразмерные области, затрудняющие или даже просто блокирующие диффузию. Достаточно представить искусственный, но вполне наглядный пример тонкой непроницаемой перегородки, разделяющей образец посередине.

Рассмотрим топологические показатели, учитывающие реально возможные пути миграции атомов пенетранта. Как видно на рис. 1 и 2, связность энергетического ландшафта (возможность пути из одной заданной точки пространства в другую) зависит от энергии частицы. Поэтому первым естественным «топологическим» показателем будет минимальная энергия, при которой атом, находящийся на одной грани образца, может достичь противоположной грани. В применении к диффузии это будет энергия активации.

Для решения такой задачи необходимо рассмотреть связные подмножества вокселей энергетического ландшафта, энергия которых не превышает некоторого порога и имеющие как минимум два вокселя, касающиеся противоположных граней расчетной ячейки. Подмножество, имеющее минимальную пороговую энергию, и будет искомым. На языке теории перколяции такая задача известна как задача построения перколяционного кластера. Для построения перколяционных кластеров, имеющих минимальную пороговую энергию, был использован широко известный волновой алгоритм (чаще используется термин «метод фронта горения» (англ. «burning method») [13], а для поиска минимума энергии - не менее известный метод дихотомии [14]. Для нивелирования анизотропии образцов, вызванных относительно малым количеством атомов матрицы (для большинства образцов - 1000 атомов кремния и 2000 атомов кислорода), кластеры строились для трех пар граней (соответственно, по оси х, у и z) и из них выбирали кластер, имеющий минимальную энергию перколяции.

Пример стереопары минимального перколяционного кластера, построенного для этого же образца, как и на рис. 1 и 2 (cr_2.200_6), показан на рис. 5. Такие минимальные перколяционные кластеры были построены для всех образцов, использованных в работе. Зависимость минимальной энергии перколяции (как уже отмечалось выше, это и есть энергия активации диффузионного процесса) от коэффициента диффузии представлена на рис. 6.

Как видно на рис. 6, образцы стекол и чисто кремниевый содалит удовлетворяют общему тренду аррениусовской зависимости. Образцы кварцевого стекла имеют разные энергии активации, но сопоставимые предэкспоненциальные множители. В то же время образец кристобалита резко выпадает из тренда, хотя имеет сопоставимую со стеклами энергию активации.

Уточняющую информацию можно получить, рассмотрев профиль потенциальной энергии вдоль пути перколяции атома гелия от одной грани ячейки к другой на траектории реакции. На рис. 7 эта траектория для перколяционного кластера образца сr_2.200_6 выделена красным. Именно по этому пути с максимальной вероятностью проходит миграция атома пенетранта.

$$
-413-
$$




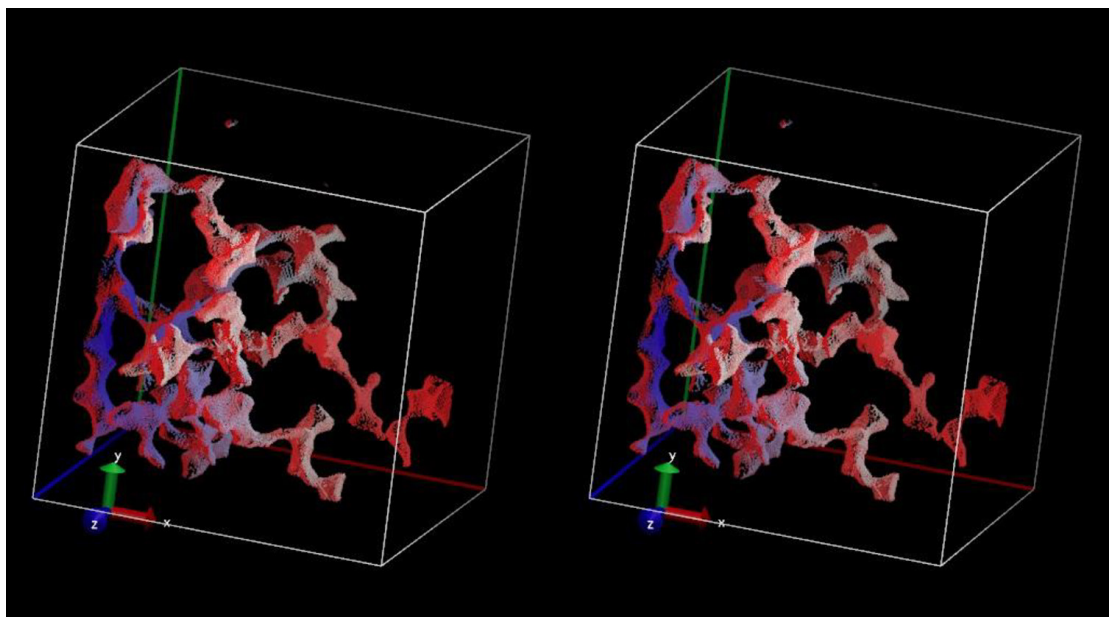

Рис. 5. Перекрестная стереопара минимального по энергии перколяционного кластера, рассчитанного по энергетическому ландшафту образца сr_2.200_6

Fig. 5. Cross stereopair of the minimum energy percolation cluster calculated from the energy landscape of the sample cr_2.200_6

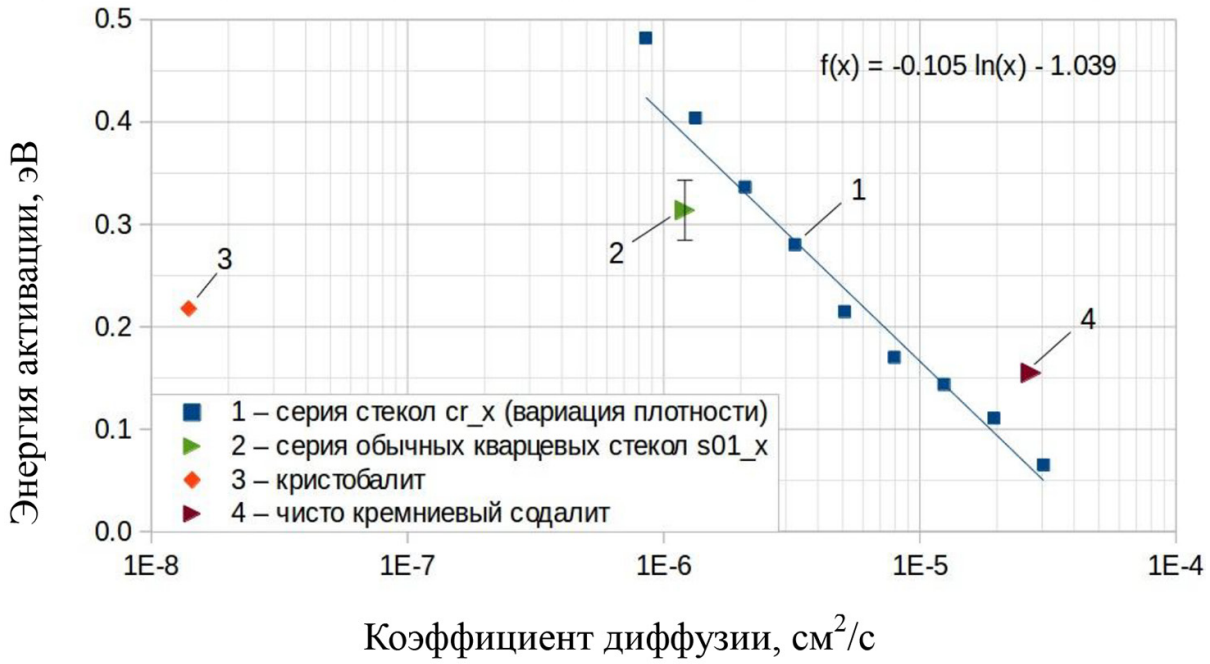

Рис. 6. Зависимость энергии активации, рассчитанная по энергетическим ландшафтам образцов, от их коэффициентов диффузии

Fig. 6. Dependence of the activation energy, calculated from the energy landscapes of the samples, on their diffusion coefficients

Профили потенциала диффундирующего атома в зависимости от относительного пути для образца сr_2.200_6 (слева) и кристобалита (справа) показаны на рис. 8. Максимумы энергии на этих кривых соответствуют седловым точкам энергетического ландшафта, а минимумы -локальным минимумам. Средние уровни энергии изображены красным. Видно, что при одинаковой плотности материала и энергии активации атому гелия в кристобалите приходится преодолевать в два раза больше потенциальных барьеров приблизительно такой же высоты (соответственно 20 и 9). Поскольку атом гелия проходит эти барьеры последовательно, то веро- 


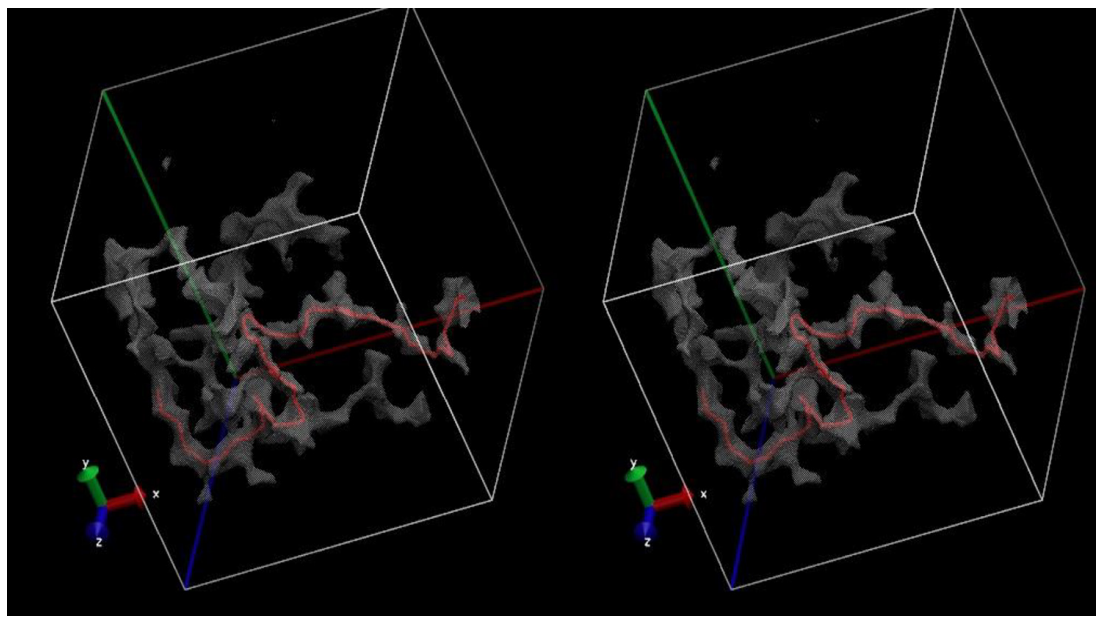

Рис. 7. Перекрестная стереопара траектории перколяции («координата реакции»)

Fig. 7. Cross stereopair percolation trajectory («reaction coordinate»)

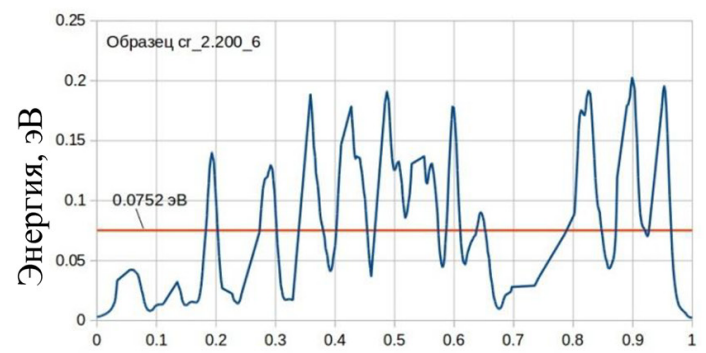

Относительный путь перколяции (безр. ед.)

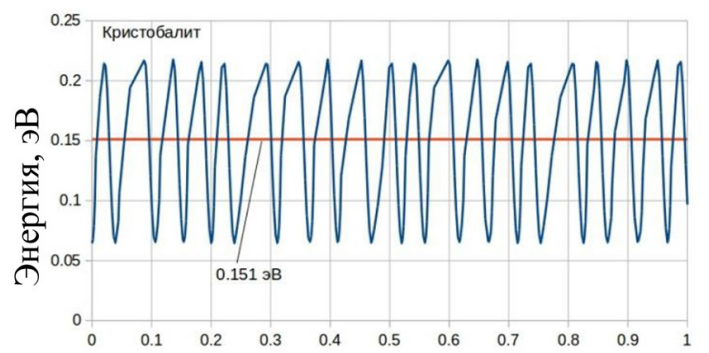

Относительный путь перколяции (безр. ед.)

Рис. 8. Профили потенциальной энергии вдоль пути перколяции для образца кварцевого стекла cr_2.200_6 (слева) и кристобалита (справа)

Fig. 8. Potential energy profiles along the percolation path for a quartz glass sample cr_2.200_6 (left) and critosbalite (right)

ятность прохождения всего образца будет пропорциональна произведению вероятностей прохождения одиночного барьера. В результате отношение коэффициентов диффузии (а в данном случае предэкспоненциальных множителей) будет пропорционально $\mathrm{p}_{0}{ }^{11}$, где $\mathrm{p}_{0}(<1)-$ вероятность прохождения одиночного барьера, а $11=20-9$ - разность в количестве этих барьеров для кристобалита и образца cr_2.200_6. Поэтому даже при сравнительно больших вероятностях это отношение может быть очень малым.

\section{Выводы}

Для поиска молекулярной структуры мембранного материала, оптимальной для заданной газовой смеси, необходимы быстрые и эффективные алгоритмы оценки диффузионных свойств модели материала. В работе показано, что трехмерный энергетический ландшафт пробной частицы пенетранта в виде трехмерного массива вокселей является эффективной структурой данных для решения этой задачи. Рассмотрен ряд показателей, легко вычисляемых по потен- 
циальным ландшафтам конкретных материалов, как не связанных с топологией потенциального ландшафта (глобальные минимумы, распределения вокселей по энергии), так и зависящих от нее (перколяционные кластеры). Показана хорошая корреляция этих показателей с соответствующими коэффициентами диффузии.

\section{Благодарности / Acknowledgements}

Исследование выполнено в рамках государственного задания Института химии и химической технологии СО РАН (проект 121031500198-3).

This work was conducted within the framework of the budget project \# 121031500198-3 for Institute of Chemistry and Chemical Technology SB RAS.

\section{Список литературы / References}

1. Sunarso J., Hashim S. S., Lin Y. S., Liu S. M. Membranes for helium recovery: An overview on the context, materials and future directions. Separation and Purification Technology 2017. Vol. 176, P. $335-383$.

2. Николаев Н.И. Диффузия в мембранах. М.: Химия, 1980. 232 с. [Nikolaev N. I. Diffusion in membranes. Moscow: Khimiya, 1980. 232 p. (In Russ.)]

3. Stern S.A., Singlair T.F., Gareis P.J. Vahldieck N.P., Mohr P.H. Helium recovery by permeation. Industrial \& Engineering Chemistry Research 1965. Vol. 57 (2), P. 49-60.

4. Robeson L. M. Correlation of separation factor versus permeability for polymeric membranes. Journal of Membrane Science 1991. Vol. 62, P. 165-185.

5. Robeson L. M. The upper bound revisited. Journal of Membrane Science 2008. Vol. 320, P. $390-400$.

6. Кухтецкий С.В. Контроль плотности кварцевого стекла в моделях, получаемых методом молекулярной динамики. Современные проблемы науки и образования 2015. № 1 . (http://www.science-education.ru/121-19349). [Kuhtetskiy S. V. Controlling the density of quartz glass in models obtained by the molecular dynamics method. Modern problems of science and education 2015. No. 1. (In Russ.)]

7. Кухтецкий С.В. Влияние обрезания потенциалов межатомных взаимодействий на структурные параметры моделей кварцевого стекла. Современные проблемы науки и образования 2015. № 2. (http://www.science-education.ru/129-22338). [Kuhtetskiy S. V. Effect of cutoff of interatomic interaction potentials on structural parameters of quartz glass models. Modern problems of science and education 2015. No. 2. (In Russ.)]

8. Kuhtetskiy S.V., Fomenko E.V., Anshits A.G. Molecular dynamics simulation of helium diffusion in vitreous silica. Journal of Non-Crystalline Solids 2016. Vol. 443, P. 47-53.

9. Wales D. Energy Landscapes: Applications to clusters, biomolecules and glasses. UK: Cambridge: Cambridge University Press, 2004. 692 p.

10. Wang C., Stratt R. M. Global perspectives on the energy landscapes of liquids, supercooled liquids, and glassy systems: Geodesic pathways through the potential energy landscape. Journal of Chemical Physics 2007, Vol. 127, P. 224504-1 - 224504-12.

11. Barrer R.M., Vaughan D.E.W. Solution and diffusion of helium and neon in tridymite and cristobalite. Transactions of the Faraday Society 1967, Vol. 63, P. 2275-2290.

$$
-416-
$$


12. Swets D.E., Lee R. W., Frank R.C. Diffusion coefficients of helium in fused quartz. Journal of Chemical Physics 1961, Vol. 34, P. 17-22.

13. Rubin F. The Lee Path Connection Algorithm. IEEE Transactions on Computers 1974, Vol. 23 (9), Р. 907-914.

14. Демидович Б.П., Марон И.А. Основы вычислительной математики. М.: Наука, 1970. 664 c. [Demidovich B. P., Maron I. A. Fundamentals of Computational Mathematics. Moscow: Nauka, 1970. 664 p. (In Russ.)] 